\title{
MYOCUTANEOUS FLAPS IN RECONSTRUCTION OF ORTHOPAEDIC DEFECTS: RURAL EXPERIENCE
}

\author{
B. C. Bakane ${ }^{1}$, Noopur Priya ${ }^{2}$, Preeti Thute ${ }^{3}$
}

\section{HOW TO CITE THIS ARTICLE:}

B. C. Bakane, Noopur Priya, Preeti Thute. "Myocutaneous Flaps in Reconstruction of Orthopaedic Defects: Rural Experience". Journal of Evolution of Medical and Dental Sciences 2015; Vol. 4, Issue 77, September 24; Page: 13531-13534, DOI: 10.14260/jemds/2015/1935

\begin{abstract}
Post traumatic and post excisional full thickness tissue defects as well as exposed implants and bones after surgery are challenging to orthopedic surgeons. Management of these complex injuries become difficult if the wound closure cannot be brought primarily. A flap is a unit of tissue that may be transferred from donor to a recipient site while maintaining its blood supply. Many such type of flaps are used to cover the defects and the donor site is either closed primarily or covered by skin graft. We present our experience of managing post traumatic, post excisional defect at Acharya Vinoba Bhave Rural Hospital by various types of flaps such as reverse sural artery flap, gastrocnemius and anterolateral thigh flaps which have significantly reduced the morbidity and long term complications of the open wounds. The non-availability of expertise, experience of surgeon in the field of reconstruction and poverty are the major constraints in rural India. Our experience of three cases is discussed.
\end{abstract}

KEYWORDS: Post excisional defect, Reverse sural artery flap, Gastrocnemius flap, Anterolateral thigh flap.

INTRODUCTION: A flap is a unit of tissue that may be transferred from a donor to a recipient site while maintaining its blood supply. The increasing interest in muscle circulation resulted in recognition of the contribution of muscle flap circulation to the overlying skin, which advanced our ability to close complex, composite defects with improved function, cosmesis, and variability within the donor options. Following debridement of the infected bone associated with chronic osteomyelitis, a muscle flap is transposed as a regional flap. The flap fills the area of bone debridement with wellvascularized tissue and provides stable wound coverage. Short-term culture-specific antibiotic therapy is used simultaneously. This approach has resulted in successful management of chronic infection in the site of bone or cartilage injury. ${ }^{1}$ in our series we are presenting cases in which orthopaedic defects created have been covered by using myocutaneous flaps.

We have used reverse sural artery flap and gastrocnemeus flap. These flaps significantly decreased the hospital stay of the patient and lead to rapid healing of wound.

Gastrocnemius Flap: Gastrocnemius flap is the choice of reconstruction of soft tissue defects in proximal $1 / 3^{\text {rd }}$ tibia and even on the knee joint. This Muscle flap have been one of the most significant development in the management of compound fractures. ${ }^{2}$ Its importance has increased specially in management of compound tibial fractures because of the poor vascularity of the region and subcutaneous nature of the bone. It consists of two bellies of gastrocnemius and involves dissection at level of neurovascular bundle.

Reverse Sural Artery Flap: This technique uses reverse flow of sural artery. This flap mainly used for reconstruction of heel and calcaneal defects. Structures involved were superficial and deep fascia, sural nerve. Lesser saphenous vein and skin. Its advantages are easy dissection, complete coverage of defect without the need of micro-vascular anastomosis. 


\section{CASE REPORT}

CASE 1: Gastrocnemius Flap: A 50 year old patient presented with fracture tibia for which she had undergone open reduction and plate osteosynthesis. Post operatively wound dehiscence and tibial plate was exposed which was reconstructed by gastocnemius flap.

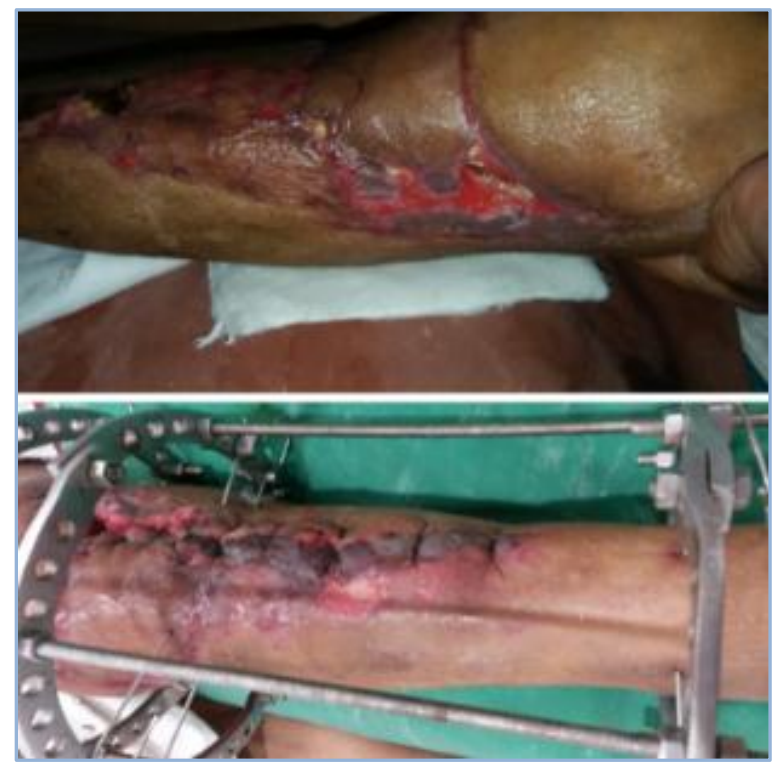

Fig. 1: Post-operative figure showing the defect covered by flap

CASE 2: Reverse Sural Artery Flap: A 35 year old female presented to us with a swelling on lateral aspect of heel. She had history of recurrence of the swelling. Wide local excision of the swelling was done which led to a calcaneal defect which was covered by reverse sural artery flap. The wound healed in a duration of 15 days and gave good cosmetic results.

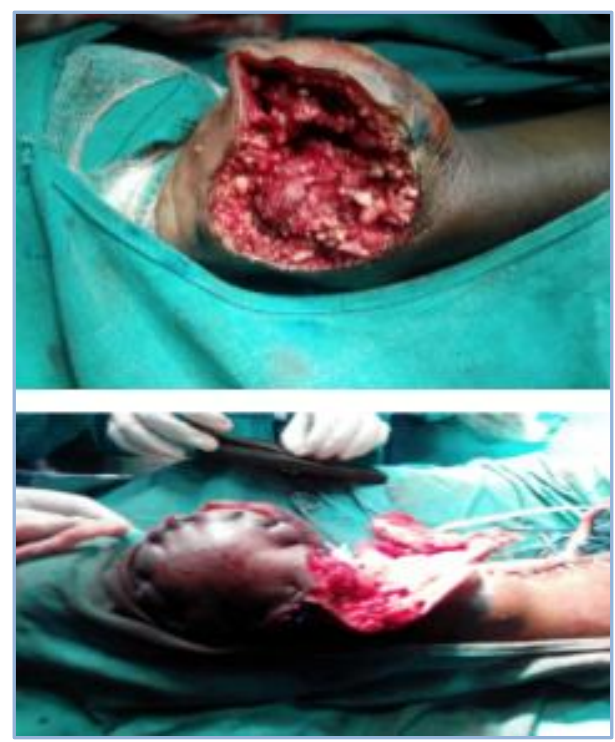

Fig. 2: Showing the Post-operative defect and coverage of defect with reverse sural artery flap 


\section{CASE REPORT}

CASE 3: Reverse Sural Artery Flap: A 30 yrs. old male presented with accidental injury to heel which was treated by primary suturing by orthopaedic surgeon. Patient presented with full thickness loss of heel pad exposing calcaneum which was reconstructed by reverse sural artery flap.
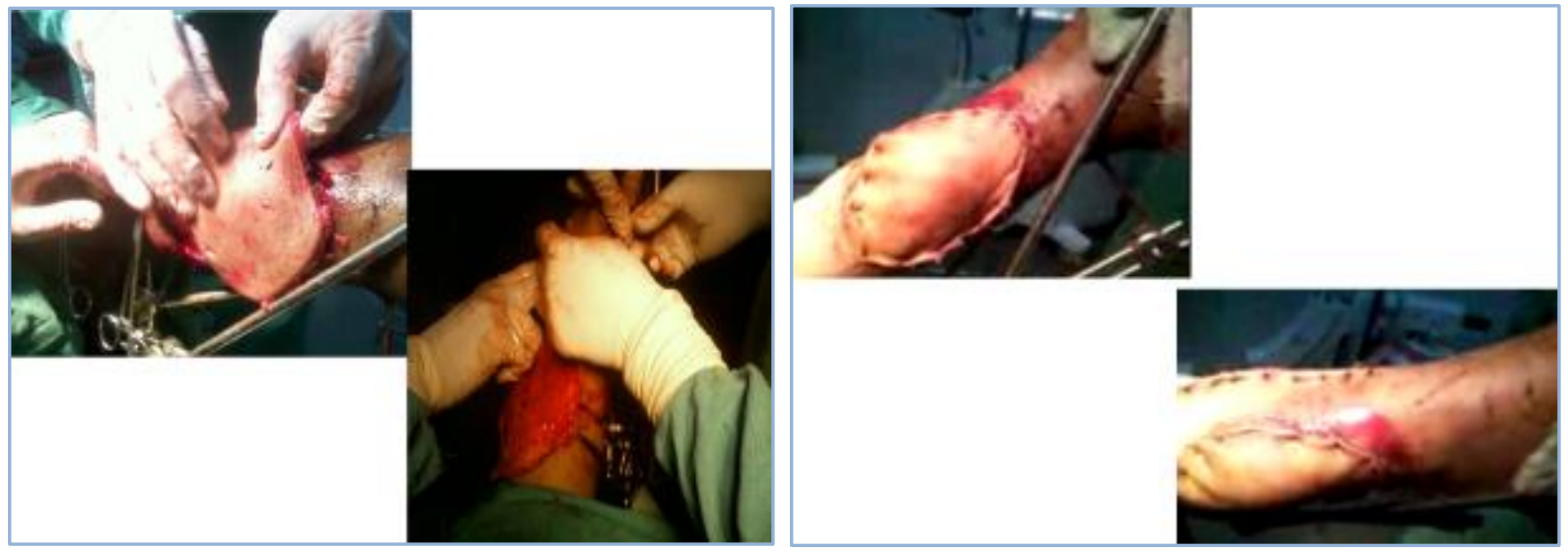

Fig. 3: Showing the intraoperative photographs of flap harvesting above and below showing the Post-operative defect closure

DISCUSSION: Muscle and musculocutaneous flaps are available in all body regions. With the selection of muscles with a suitable vascular pedicle, the muscle may be safely elevated to provide coverage and simultaneously restore form and function. Reconstructive procedures are frequently required to cover the exposed bones/joints, obliterate the dead space, and help eradicate the infection of chronic osteomyelitis and prepare and vascularize the wound for subsequent bone grafting. ${ }^{3}$

Muscle flaps are also suitable for coverage of open joints and exposed orthopaedic implants. ${ }^{4}$ early cover has been found to considerably reduce the incidence of complications. Use of muscle and musculocutaneous flaps broadens the options for defect closure in every area of the body. Even big defects like calcaneal defects, trochanteric defects and tibial defects can be closed by myocutaneous flaps. Muscle flaps, by virtue of their excellent intrinsic blood supply and mouldable nature that fills in the irregular cavities of the bone, are the best solution for such defects. ${ }^{5}$ this technique is useful as no mortality and morbidity has been observed.

CONCLUSIONS: The cases in our series with the use of myocutaneus flaps have given good results in terms of cosmesis and functional ability of the patient. The defect healed in a lesser time and resulted in decreased hospital stay of the pateint. Post op complications like hematoma, infections, osteomyletis were not reported in any of the cases No mortality was observed.

\section{REFERENCES:}

1. Mathes SJ, Alpert BS, Chang N. Use of the muscle flap in chronic osteomyelitis: experimental and clinical correlation. Plast Reconstr Surg. 1982; 69:185.

2. Vasconez LO, Bostwick J, McCraw JB. Coverage of exposed bone by Muscle transposition and skin grafting. Plastic Reconstr. Surg. 1974; 53: 526-530.

3. Mathes SJ, Nahai F. Clinical applications for muscle and musculocutaneous flaps. New York; CV Mosby Company Publishers. 1982: 16-26, 514-524. 


\section{CASE REPORT}

4. Byrd HS, Spicer TE., Cierney G. Management of open tibial fractures. Plastic Reconstr Surg 1985; 76: 719-730.

5. Meller I, Ariche A, Sagi A. The role of gastrocnemius muscle flap in limb sparing surgery for bone sarcomas of the distal femur: A proposed classification of muscle transfers. Plastic Reconstr Surg 1997; 99: 751-756.

\section{AUTHORS:}

1. B. C. Bakane

2. Noopur Priya

3. Preeti Thute

\section{PARTICULARS OF CONTRIBUTORS:}

1. Professor, Department of General Surgery, MMC Acharya Vinoba Bhave Rural Hospital.

2. Resident, Department of General Surgery, MMC Acharya Vinoba Bhave Rural Hospital.

3. Associate Professor, Department of Anatomy, MMC Acharya Vinoba Bhave Rural Hospital.

FINANCIAL OR OTHER COMPETING INTERESTS: None
NAME ADDRESS EMAIL ID OF THE CORRESPONDING AUTHOR:

Dr. B. C. Bakane,

Professor,

Department of General Surgery, MMC Acharya Vinoba Bhave

Rural Hospital, Sawangi, Meghe, Wardha-442001.

E-mail: b_bakane@rediffmail.com

Date of Submission: 02/09/2015.

Date of Peer Review: 03/09/2015.

Date of Acceptance: 18/09/2015.

Date of Publishing: 24/09/2015. 